Original article

\title{
Cryptococcal transmigration across a model brain blood-barrier: evidence of the Trojan horse mechanism and differences between Cryptococcus neoformans var. grubii strain H99 and Cryptococcus gattii strain R265
}

Tania C. Sorrell ${ }^{\mathrm{a}, *}$, Pierre Juillard ${ }^{\mathrm{a}, \mathrm{b}, \mathrm{d}}$, Julianne T. Djordjevic ${ }^{\mathrm{a}, \mathrm{b}}$, Keren Francis ${ }^{\mathrm{a}, \mathrm{b}}$, Anelia Dietmann ${ }^{\mathrm{c}, \mathrm{d}}$, Alban Milonig ${ }^{\mathrm{c}, \mathrm{d}}$, Valery Combes ${ }^{\mathrm{a}, \mathrm{d}}$, Georges E.R. Grau ${ }^{\mathrm{a}, \mathrm{d}}$

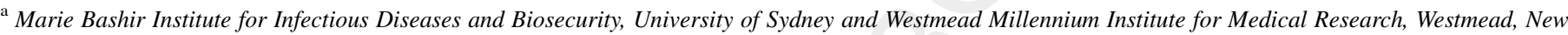 \\ South Wales 2145, Australia \\ ${ }^{\mathrm{b}}$ Fungal Pathogenesis Laboratory, Centre for Infectious Diseases and Microbiology, Westmead Millennium Institute, Westmead 2145, Australia \\ ${ }^{\mathrm{c}}$ Department of Neurology, Innsbruck Medical University, Innsbruck, Austria \\ ${ }^{\mathrm{d}}$ Vascular Immunology Unit, Department of Pathology, School of Medical Sciences, University of Sydney, NSW 2006, Australia
}

Received 11 May 2015; accepted 31 August 2015

Available online $\mathbf{a} \mathbf{a}$

\begin{abstract}
Cryptococcus neoformans $(\mathrm{Cn})$ and Cryptococcus gattii $(\mathrm{Cg})$ cause neurological disease and cross the BBB as free cells or in mononuclear phagocytes via the Trojan horse mechanism, although evidence for the latter is indirect. There is emerging evidence that $C n$ and the North American outbreak $C g$ strain (R265) more commonly cause neurological and lung disease, respectively. We have employed a widely validated in vitro model of the $\mathrm{BBB}$, which utilizes the hCMEC/D3 cell line derived from human brain endothelial cells (HBEC) and the human macrophage-like cell line, THP-1, to investigate whether transport of dual fluorescence-labelled $C n$ and $C g$ across the BBB occurs within macrophages. We showed that phagocytosis of $C n$ by non-interferon (IFN)- $\gamma$ stimulated THP-1 cells was higher than that of $C g$. Although $C n$ and $C g$-loaded THP-1 bound similarly to TNF-activated HBECs under shear stress, more $C n$-loaded macrophages were transported across an intact HBEC monolayer, consistent with the predilection of $C n$ for CNS infection. Furthermore, $C n$ exhibited a higher rate of expulsion from transmigrated THP-1 compared with $C g$. Our results therefore provide further evidence for transmigration of both $C n$ and $C g$ via the Trojan horse mechanism and a potential explanation for the predilection of $C n$ to cause CNS infection.
\end{abstract}

(C) 2015 Institut Pasteur. Published by Elsevier Masson SAS. All rights reserved.

Keywords: Cryptococcus; Monocytes; Brain endothelium; Phagocytosis; Transmigration

\section{Introduction}

Despite the known predilection of $C n$ and $C g$ for the lung and central nervous system, the emergence of the VGII

\footnotetext{
* Corresponding author. Marie Bashir Institute for Infectious Diseases \& Biosecurity, University of Sydney; Centre for Infectious Diseases and Microbiology, Westmead Millennium Institute, 178 Hawkesbury Rd, WestQ1 mead NSW 2145, Australia. Tel.: +61 298456029.

E-mail address: tania.sorrell@sydney.edu.au (T.C. Sorrell).
}

genotype of Cryptococcus gattii on Vancouver Island several years ago has revealed differences in clinical presentation [1]. In addition, $C n$ and $C g$ differ in host preference [2]. For example, $C g$ VGII most commonly causes lung disease and affects apparently healthy hosts whereas $C n$ var. grubii (molecular types VNI and VNII predominantly) typically causes meningitis in HIV-infected or otherwise immunecompromised hosts. In rat and mouse models, infection via the respiratory route with the type strain of $\mathrm{Cn}, \mathrm{H} 99$ and the VGII outbreak strain of $C g$ (R265), resulted in mortality due 
to neurological and lung infection, respectively $[3,4]$. The genesis of these differences is unexplained but may involve differential passage across the blood brain barrier (BBB). This hypothesis is consistent with the work of Ngamskulrungroj et al. [4], who demonstrated that the load of cryptococci in the brains of mice injected intravenously with $\mathrm{Cn}$ strain $\mathrm{H} 99$ was higher than that in mice infected with R265, and that small inocula given intravenously resulted in much higher mortality in the group receiving H99.

Cryptococci cause neurological disease following dissemination from the lung via the blood and passage across the blood brain barrier (BBB). Studies in murine models of $\mathrm{Cn}$ infection suggest that cryptococci enter the brain through the microvasculature of cortical and penetrating vessels $[5,6]$, form para-vascular micro-abscesses and spread secondarily to the meninges, with development of meningitis. Debate has centered on whether cryptococci are transported in the blood and/or across the BBB as free cells or within mononuclear phagocytes (the so-called Trojan horse mechanism). In animal and cell models of cryptococcosis, it has been shown that free cryptococci can either cross the BBB via specific ligandreceptor interactions followed by transcytosis $[7,8]$ or via the mechanical trapping of cryptococci in the branches of the capillaries followed by mechanical and/or biochemical disruption of the capillary walls of endothelial cells and/or their tight junctions (e.g. following exposure to cryptococcal urease) $[9,10]$. Cryptococci have also been found in the blood of experimental animals in association with monocytes $[11,12]$ and there is indirect evidence for transmigration across the BBB by the Trojan horse mechanism. Specifically, (a) adoptive transfer of pulmonary macrophages and blood monocytes isolated from mice infected with $\mathrm{Cn}$ via inhalation, led to cerebral cryptococcosis in healthy recipients [11]; (b) intravenous inoculation of murine macrophages infected with cryptococci in vitro resulted in meningo-encephalitis, and (c) clodronate-induced depletion of circulating monocytes reduced disease severity and tissue fungal burdens [5] [12]. In the context of AIDS-associated cryptococcal meningo-encephalitis, it is notable that mononuclear phagocyte translocation into the CNS is facilitated by HIV infection of these cells [13]. Demonstration of non-lytic exocytosis, whereby cryptococci are expelled from mononuclear phagocytes without affecting the viability of either cell type $[14,15]$ raises the additional possibility that they are transported via the blood stream within monocytes but are expelled following cellular adherence to cerebral microvascular endothelial cells and thence cross the BBB as free cryptococci.

In this study we employed a widely validated in vitro model of the BBB, which utilizes the hCMEC/D3 cell line derived from human brain endothelial cells (HBEC), which recapitulates functions and cell surface properties human endothelial cells of the brain microvasculature [16], and the human macrophage-like cell line, THP-1, to demonstrate the existence of the Trojan horse mechanism of transport of $\mathrm{Cn}$ and $C g$ across the BBB and to investigate species-dependent differences in this process.

\section{Materials \& methods}

\subsection{Endothelial and monocyte cell lines}

Human cerebral microvascular endothelial cells (hCMEC/ D3), referred to as HBEC, were a generous gift from Professor P.O. Couraud, (Hopital Cochin, Paris, France). THP-1 monocyte-like cells of human origin were obtained from Dr B. Saunders (Centenary Institute, Sydney, Australia) [17].

\subsection{Cryptococcal strains}

Cn strain H99 (originally a gift of Dr Gary Cox, Duke University) and $C g$ strain R265 (the more virulent genotype VGII originating from a patient in the Vancouver Island outbreak) - from the collection of W. Meyer, Westmead, NSW, Australia) were sub-cultured from glycerol stocks and cultured at $30{ }^{\circ} \mathrm{C}$ overnight on yeast-peptone-dextrose (YPD: USB, Cleveland, Ohio, USA) agar prior to use.

\subsection{FITC labelling and opsonisation of cryptococci}

Cryptococci were pelleted from overnight YPD cultures, washed with PBS, labelled with FITC in PBS (Sigma Aldrich, St Louis, MO, USA) by incubation at $30{ }^{\circ} \mathrm{C}$ for $10 \mathrm{~min}$ with light agitation, pelleted by centrifugation and washed $3 \times$ with Dulbecco's PBS (Lonza, Basel, Switzerland) or until the supernatant was clear. Labelled cryptococci were counted in a haemocytometer and a volume containing $15 \times 10^{6}$ cells was centrifuged. The pellet was resuspended in $750 \mu \mathrm{L}$ human serum prepared from clotted blood and $3.75 \mu \mathrm{L} \mathrm{IgG} \mathrm{opso-}$ nising antibody (18B7) directed against the cryptococcal capsular polysaccharide, glucuronoxylomannan (GXM), a kind gift from Arturo Casadevall, Albert Einstein College of Medicine, Bronx, New York). Cryptococci were incubated in serum and antibody for $30 \mathrm{~min}$ in a rotary shaker with light agitation at $37{ }^{\circ} \mathrm{C}$ and pelleted by centrifugation. $150 \mu \mathrm{L}$ of serum/antibody supernatant was removed to bring the final volume of supernatant to $600 \mu \mathrm{l}$. Cryptococci were resuspended in this supernatant before further use.

\subsection{Phagocytosis of cryptococci by THP-1 cells, calculation of phagocytic index and analysis by flow cytometry}

THP-1 cells were cultured in RPMI complete medium and were either left unstimulated or stimulated overnight with IFN- $\gamma\left(10 \mathrm{ng} / \mathrm{mL}\right.$, Peprotech, Rocky Hill NJ USA) at $37^{\circ} \mathrm{C}$ in a $5 \% \mathrm{CO}_{2}$ atmosphere. Cryptococci were then labelled with FITC and opsonized as described above. IFN- $\gamma$ stimulated and non-stimulated (NS) THP-1 cells were resuspended in RPMI medium and FITC-labelled, opsonized cryptococci were added in a ratio of 1:5 THP-1 cells:cryptococci. Phagocytosis was allowed to proceed for $3 \mathrm{~h}$ at $37{ }^{\circ} \mathrm{C}$ in a $5 \% \mathrm{CO}_{2}$ atmosphere. After incubation, suspensions were washed by centrifugation and labelled with $1.5 \mathrm{mM}$ Uvitex solution (Fluorescent Brightener 28, Sigma F-3543) for $10 \mathrm{~min}$ at 
$37{ }^{\circ} \mathrm{C}$ with light agitation. Images were captured and deconvoluted using a DeltaVision Deconvolution microscope.

To calculate the phagocytic index, a minimum of 100 THP1 cells were counted per treatment in triplicates, along with the number of cryptoccoci ingested per THP-1 cell. Phagocytic index (PI), was determined using the following calculation: $\mathrm{PI}=(\%$ phagocytic cells containing $\geq$ cryptococci $) \times($ mean number of cryptococci/phagocytic cell containing cryptococci).

For flow cytometry analysis, the pellets of both stimulated and non-stimulated THP-1, incubated with either $C n$ or $C g$ were resuspended in RPMI and aliquoted into a round-bottom 96 well plate. Samples were analysed on a Cytomics FC500 MPL or a Gallios (Beckman Coulter) flow cytometer (Beckman Coulter, Indianapolis, IN, USA).

\subsection{Binding under shear stress}

\subsubsection{Preparation of HBEC monolayers}

HBEC were seeded on to collagen-coated $35 \mathrm{~mm}$ culture dishes (Corning, NY, USA, Cat \# 430165), grown to confluence in EBM2 medium as previously described (14) then stimulated overnight with TNF to simulate inflammatory conditions and up-regulate adhesion molecules $(10 \mathrm{ng} / \mathrm{mL}$; Peprotech).

\subsubsection{Preparation of THP-1 cells}

Control and IFN- $\gamma$-stimulated THP-1 cells, prepared as described above, were centrifuged at $500 \mathrm{~g}$ for $5 \mathrm{~min}$ at room temperature and labelled with Wheat Germ Agglutinin (WGA, Life Technologies, Grand Island, NY, USA), at a final concentration of $1 \mu \mathrm{g} / \mathrm{mL}$ in RPMI, for $10 \mathrm{~min}$ in the dark at $37^{\circ} \mathrm{C}$. WGA is a lipophilic fluorescent dye that is very quickly incorporated into the THP-1 cell plasma membrane. Cells were washed twice, resuspended in RPMI complete medium and incubated with FITC-labelled, opsonized cryptococci for $3 \mathrm{~h}$ as described above. Post phagocytosis, cells were washed in complete medium, their numbers adjusted to $2 \times 10^{5}$ cells/ $\mathrm{ml}$ and cultures were maintained at $37{ }^{\circ} \mathrm{C}$ for the duration of the flow binding assay.

\subsubsection{Assessment of binding under flow conditions}

The flow 24 chamber apparatus consisted of a $0.010 \mathrm{~mm}$ Gasket with a $5.0 \mathrm{~mm}$ wide channel (GlycoTech, Gaithersburg, MD, USA) attached to a cast acrylic flow deck. A syringe pump (KD Scientific, Holliston, MA, USA) and a vacuum source were connected to each end of the flow 24 chamber. With the vacuum on, the flow chamber apparatus was then lowered onto a $35 \mathrm{~mm}$ petri dish containing confluent endothelium in resting or stimulated states, which was seated onto the stage of an inverted microscope (Olympus, IX71). Once a complete vacuum was established, RPMI complete medium was allowed to flow through the connecting tubing for 3 min to prevent air bubbles from interfering with the flow of cells. Approximately $3.5 \mathrm{ml}$ of THP-1 cell suspension $\left(2 \times 10^{5}\right.$ cells $\left./ \mathrm{ml}\right)$ was then loaded into the $5 \mathrm{ml}$ syringe connected to the input tubing. A flow rate of $0.227 \mathrm{ml} / \mathrm{min}$ (corresponding to 1 dyne $/ \mathrm{cm}^{2}$ or $0.1 \mathrm{~Pa}$ ) was applied for 15 min to allow cell attachment to the HBEC monolayer. Prior to counting the bound cells the monolayer was washed with complete medium for $3 \mathrm{~min}$, or until no cells were observed in the flow-through. Ten randomly selected fields of view were imaged per test condition at $200 \times$ magnification. Images of WGA-stained THP-1 cells (red) and FITC-stained cryptococci (green) were captured. Images were then overlaid to determine the proportion of bound THP-1 cells containing cryptococci (yellow). Overlain images were also used to distinguish between phagocytosed cryptococci and non-internalized (adherent) yeast. These images were imported into Image $\mathbf{J}$ analysis software and analysed using the cell counter plug-in.

\subsection{Transmigration}

\subsubsection{Endothelial cell monolayers}

Corning $6.5 \mathrm{~mm}$ and $24 \mathrm{~mm}$ Transwells with $8 \mu \mathrm{m}$ pores (Corning, NY, USA) were coated with $3 \%$ collagen. Each insert was rinsed with EBM2 medium, and $600 \mu \mathrm{l}$ and $2.6 \mathrm{ml}$ of EBM2 was added to the bottom chamber of the transwell, respectively. HBEC were seeded at $2 \times 10^{4}$ and $2 \times 10^{5}$ cells/ well, respectively, and grown to confluence at $37^{\circ} \mathrm{C}$ in an atmosphere of $5 \% \mathrm{CO}_{2}$, as determined using a sentinel well stained with WGA $(5 \mu \mathrm{g} / \mathrm{ml})$. FITC-labelled, opsonized cryptococci were allowed to undergo phagocytosis by THP1 cells as described above. Free extracellular cryptococci were removed by two rounds of centrifugation over Ficoll-paque (GE Healthcare, Uppsala, Sweden). THP-1 cells containing phagocytosed or adherent cryptococci were collected from the interface and stained with $1.5 \mu \mathrm{M}$ Uvitex (Fluorescent Brightener 28, Sigma F-3543) for $10 \mathrm{~min}$ in a rotary shaker with light agitation at $37^{\circ} \mathrm{C}$. Since Uvitex stains chitin in the fungal cell wall and does not penetrate through membranes of viable THP-1 cells, it enables cryptococci that have crossed the endothelial monolayer as free yeasts to be distinguished from those that have crossed inside THP-1 cells and been released subsequently. Suspensions of THP1 cells \pm cryptococci $\left(3 \times 10^{5}\right.$ in $100 \mathrm{uL}$ and $2.4 \times 10^{6}$ THP- 1 in $1.5 \mathrm{ml}$ ) were added to HBEC monolayers in the small and large Transwells, respectively. After overnight incubation, medium and cells within the lower chamber of the Transwell were collected, centrifuged and imaged by DeltaVision deconvolution microscope or analysed by flow cytometry.

\subsection{Effects of transmigration on the endothelial monolayer integrity}

\subsubsection{Impedance studies}

Electrode arrays (Applied BioPhysics, Troy, New York) were pre-treated with L-cysteine (10 $\mathrm{mM}$, Sigma) for $15 \mathrm{~min}$, washed twice in sterile water and then coated with $3 \%$ collagen for $1 \mathrm{~h}$. HBEC were seeded at $1 \times 10^{5} \mathrm{cells} / \mathrm{ml}$ and loaded into the electrical cell-substrate impedance sensing (ECIS) morphological biosensor (Applied Biophysics) at $37{ }^{\circ} \mathrm{C}$ for a minimum of $48 \mathrm{~h}$ or until confluence was attained (plateau). Resting and IFN $\gamma$-stimulated THP-1 that had 
phagocytosed cryptococci ( $\mathrm{Cn}$ and $\mathrm{Cg}$ ) were submitted to 2 rounds of centrifugation through Ficoll-paque to eliminate free cryptococci, and then added to the resting or TNF-stimulated HBEC at a ratio of 1 HBEC to 3 THP- 1 cells $\pm C n$ or $\mathrm{Cg}$. Impedance readings of the endothelial monolayer were taken at $5 \mathrm{~min}$ intervals for $96 \mathrm{~h}$. As controls, medium or THP-1 alone were added to separate wells.

\subsubsection{Measurement of $70 \mathrm{kDa}$ dextran permeability}

Integrity of the HBEC monolayer was also assessed by measuring its permeability to dextran. HBEC were seeded onto $8 \mu \mathrm{m}$ pore size, collagen-coated Transwell inserts in 24 well plates at $2 \times 10^{5}$ cells $/ \mathrm{ml}$ and grown until confluent. Resting THP-1 cells that had phagocytosed cryptococci $(\mathrm{Cn}$ and $\mathrm{Cg}$ ) were submitted to 2 rounds of centrifugation through Ficoll-paque to eliminate free cryptococci and then incubated for $24 \mathrm{~h}$ with the non-stimulated HBEC at a ratio of $1 \mathrm{HBEC}$ to 3 THP-1 cells \pm cryptococci $(C n$ or $C g$ ). A 30 min treatment of the endothelial monolayer with cytochalasin D (10 $\mu \mathrm{g} /$ $\mathrm{mL}$ ) was used as positive control for loss of monolayer integrity. After $24 \mathrm{~h}$, the culture medium in the upper chamber was replaced with $70 \mathrm{kDa}$ FITC-dextran $(1 \mathrm{mg} / \mathrm{ml}$, Invitrogen) diluted in DMEM without phenol red (Gibco). The lower chamber was filled with $600 \mu \mathrm{l}$ of DMEM without phenol red. After gentle resuspension, $50 \mu \mathrm{l}$ samples were removed from the lower chamber after $0,30,60,90$ and $120 \mathrm{~min}$ and the fluorescence intensity measured on a Fluostar Optima (BMG Labtech).

\subsubsection{Visualisation of the integrity of the monolayer after assessment of electrical resistance}

At the end of the ECIS experiment, the monolayers were washed gently to remove the unbound THP-1 cells and observed using phase contrast microscopy.

\subsection{Statistical analysis}

Statistical analysis was performed using either GraphPad Prism software version 5.0. or JMP statistical software (SAS Institute, Inc., Cary, NC). Comparisons of discrete or continues probability distribution between phagocytosed $\mathrm{Cn}$ and $\mathrm{Cg}$, or transmigrated $\mathrm{Cn}$ and $\mathrm{Cg}$ were analysed by using a oneway ANOVA test. A probability (p) value of $<0.05$ was considered to be significant.

\section{Results}

\subsection{Phagocytosis of $\mathrm{Cn}$ and $\mathrm{Cg}$ by THP-1 cells}

We first compared the ability of un-stimulated and IFN- $\gamma$ stimulated THP-1 cells to phagocytose $C n$ and $C g$ using flow cytometry. Control THP-1 cells (Fig. 1A, red ellipse) were easily distinguished from THP-1 cells that had phagocytosed $C n$ or $C g$ (Fig. 1B, black ellipse) or free cryptococci (Fig. 1B, green ellipse) on the basis of both forward scatter and FITC fluorescence. Flow cytometry profiles were similar regardless of the activation status of the THP- 1 cells or the cryptococcal species (not shown). After the 3-h incubation, When expressed as the proportion of THP-1 cells which had ingested at least one cryptococcal cell, phagocytosis of $\mathrm{Cn}$ and of $\mathrm{Cg}$ was significantly increased by activation with IFN- $\gamma$ and for both conditions phagocytosis of $\mathrm{Cn}$ was significantly higher than $C g$ (Fig. 1C). The load of $C n$ and $C g$ per THP-1 cell was similarly increased by IFN- $\gamma$ activation, with the load in unstimulated cells being significantly higher for $\mathrm{Cn}$ than $\mathrm{Cg}$ (Fig. 1D). The respective phagocytic indices (PI) are shown in Fig. 1D. Phagocytosis in the absence of opsonisation was minimal, whether or not THP-1 cells had been treated with $\mathrm{IFN}-\gamma$ (not shown).

\subsection{Binding to brain endothelial cells under flow conditions}

Binding of post-phagocytic THP-1 cells to HBEC was evaluated under flow conditions $\left(1 \mathrm{dyne} / \mathrm{cm}^{2}\right)$ to determine if there were differences between THP-1 cells that had been preincubated with FITC-labelled $C n$ and $C g$. In the case of resting endothelium, no significant difference between the low level of binding of $\mathrm{Cn}$ - or $\mathrm{Cg}$-loaded, non-stimulated and IFN$\gamma$-stimulated THP-1 cells was observed, hence only the results for non-stimulated THP-1 cells are shown. In contrast, stimulation of the HBEC monolayer with TNF greatly enhanced binding of both $C n$ and $C g$-loaded THP-1 cells, regardless of the activation status of the THP-1 cells (Fig. 2 A and B, mid and lower histograms). For binding of cells pre-incubated with $C n, 17 \pm 5$ THP- 1 cells per 10 fields of view (FOV) adhered to the resting monolayer with $4 \pm 2(21 \%)$ containing $\mathrm{Cn}$ (Fig. 2A). This number increased to $269 \pm 42$, with $175 \pm 8$ cells $(65 \%$, black bar) containing $C n$ after the endothelial monolayer had been stimulated with TNF (Fig. 2A). Pre-incubation of THP-1 cells overnight with IFN- $\gamma$ did not significantly increase the number bound to TNFstimulated HBEC $(285 \pm 95.1$, with $181 \pm 38.5$ cells containing $C n$ (64\%, black bar). A representative micrograph distinguishing the binding of empty THP-1 cells (red) from those containing intracellular $C n$ (green or yellow) to TNFstimulated HBEC is shown in Fig. 2D. No binding of either empty or loaded THP-1 cells to resting HBEC is evident in Fig. 2C.

As with $C n$, limited binding of $C g$-containing THP- 1 cells to non-stimulated HBEC was observed: $20 \pm 4.2$ THP- 1 cells were bound, with $3 \pm 1.4$ (15\%) containing $C g$ (Fig. 2B, upper bar). As observed for $C n$-loaded THP-1 cells in Fig. 2A, there was no significant difference between the binding of nonstimulated and IFN- $\gamma$-stimulated, pre-loaded THP-1 cells to resting endothelium and thus only the results for nonstimulated THP-1 cells are shown. When HBEC were activated with TNF, the total number of bound, non-stimulated, pre-loaded THP-1 cells increased to $487 \pm 304.1$, with $275 \pm 167$ (57\%, black bar) containing $C g$ (Fig. 2B). Stimulation with IFN- $\gamma$ did not change the binding of the $C g$-loaded THP- 1 cells to HBEC: $508 \pm 232.9$ THP-1 cells were bound to the monolayer, with $300 \pm 179.9$ cells $(59 \%$, black bars) containing $\mathrm{Cg}$ (Fig. 2B). 
A

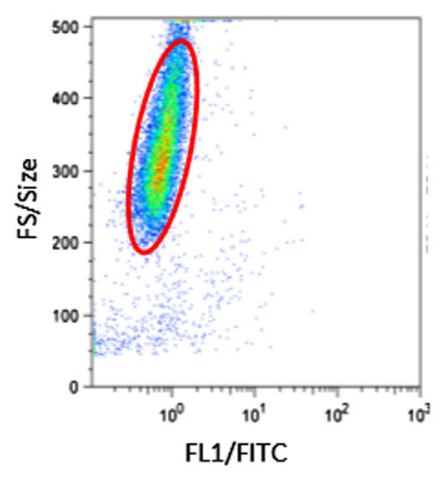

B

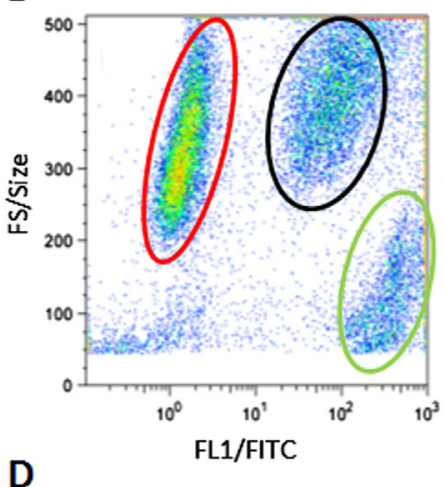

66

67

68

69

Fig. 1. Differential phagocytosis of $\boldsymbol{C}$ and $\boldsymbol{C g}$ by the human monocytic cell line, THP-1. (A, B): Flow cytometric profiles of THP-1 cells only (A, red gate) and with phagocytosed FITC-positive cryptococci (B, black gate). Free FITC-positive cryptococci are present in the green gate (B). (C) Non-stimulated (NS) or IFN- $\gamma$ stimulated THP-1 cells were co-cultured with opsonised, FITC-labelled cryptococci for $3 \mathrm{~h}$, and the extent of phagocytosis assessed by flow cytometry by gating on the FITC-positive THP-1 cell population (black gate, B). Five separate experiments were performed. The extent of cryptococcal phagocytosis under the various conditions is expressed as a ratio relative to the phagocytosis of $C n$ by unstimulated THP-1 $(70.3 \pm 3.4 \%)$. Phagocytosis of $C n$ by unstimulated THP-1 cells was significantly greater than that of $C g$. (D) Cryptococcal-phagocytic activity. Number of phagocytosed cryptococci in non-stimulated and INF- $\gamma$ stimulated THP1 cells. X marks the minimum and maximum number of engulfed cryptococci per THP-1 cell. PI - Phagocytic Index, as defined by the percentage of phagocytic THP-1 cells containing $\geq 1$ cryptococcal cell multiplied by the mean number of cryptococci/phagocytic cell containing cryptococci, was calculated and appears beneath the $\mathrm{X}$-axis in Fig. 1D.

\subsection{Assessment of crossing the BBB via a Trojan horse mechanism}

Transmigration experiments using FITC-labelled $\mathrm{Cn}$ and fluorescence microscopy were performed to obtain evidence that THP-1 cells transport cryptococci through the in vitro BBB model (Fig. 3). An Uvitex staining step was employed post-phagocytosis and pre-transmigration to label any nonphagocytosed cryptococci, including those that adhered to the THP-1 cell surface (FITC-positive and Uvitex-positive). This step was essential to distinguish these non-internalised from internalized (FITC-positive and Uvitex-negative) cryptococci, pre-transmigration. Fig. 3A shows dual-stained cryptococci immediately after phagocytosis showing green (intracellular) and blue or blue-green (extracellular) cryptococci. Note: these images were prepared prior to removal of extracellular cryptococci by two centrifugation steps through ficoll-hypaque, in order to demonstrate both intracellular and extracellular cryptococci. Post-transmigration, the presence of green FITC-positive, Uvitex-negative cryptococci is indicative of cryptococcal transport across the endothelial cell monolayer within THP-1 (Fig. 3B). These images clearly demonstrate transmigration of $C n$ and $C g$ via the Trojan horse mechanism.

\subsection{Comparing the transmigration of $\mathrm{Cn}$ and $\mathrm{Cg}$}

Using the dual-staining procedure described above, the transmigration of $\mathrm{Cn}$ and $\mathrm{Cg}$ via the Trojan horse mechanism was compared using both flow cytometry and fluorescence microscopy. Flow cytometry was used initially to determine the total numbers of THP-1 cells that had transmigrated into the baso-lateral chamber (Fig. 4). Fluorescence microscopy was then employed to distinguish the different sub-populations present, based on the dual staining procedure. Initial experiments (in triplicate) revealed that transmigration of THP1 cells post exposure to cryptococci was unaffected by TNF activation or not of HBEC monolayers of by activation of THP-1 cells. Results for transmigration across unstimulated HBEC are depicted in Fig. 4 After 24 h, 231,176 $\pm 125,208$ THP-1 cells had transmigrated, with $7617(3.1 \%)$ of them containing $C n$ and thus FITC-positive (Fig. 4A, white histogram). Among these, 5839 THP-1 (77\%) had transmigrated within THP-1 cells as they were Uvitex-negative (black 
A

Total THP-1 cells in $10 \mathrm{FOV}$
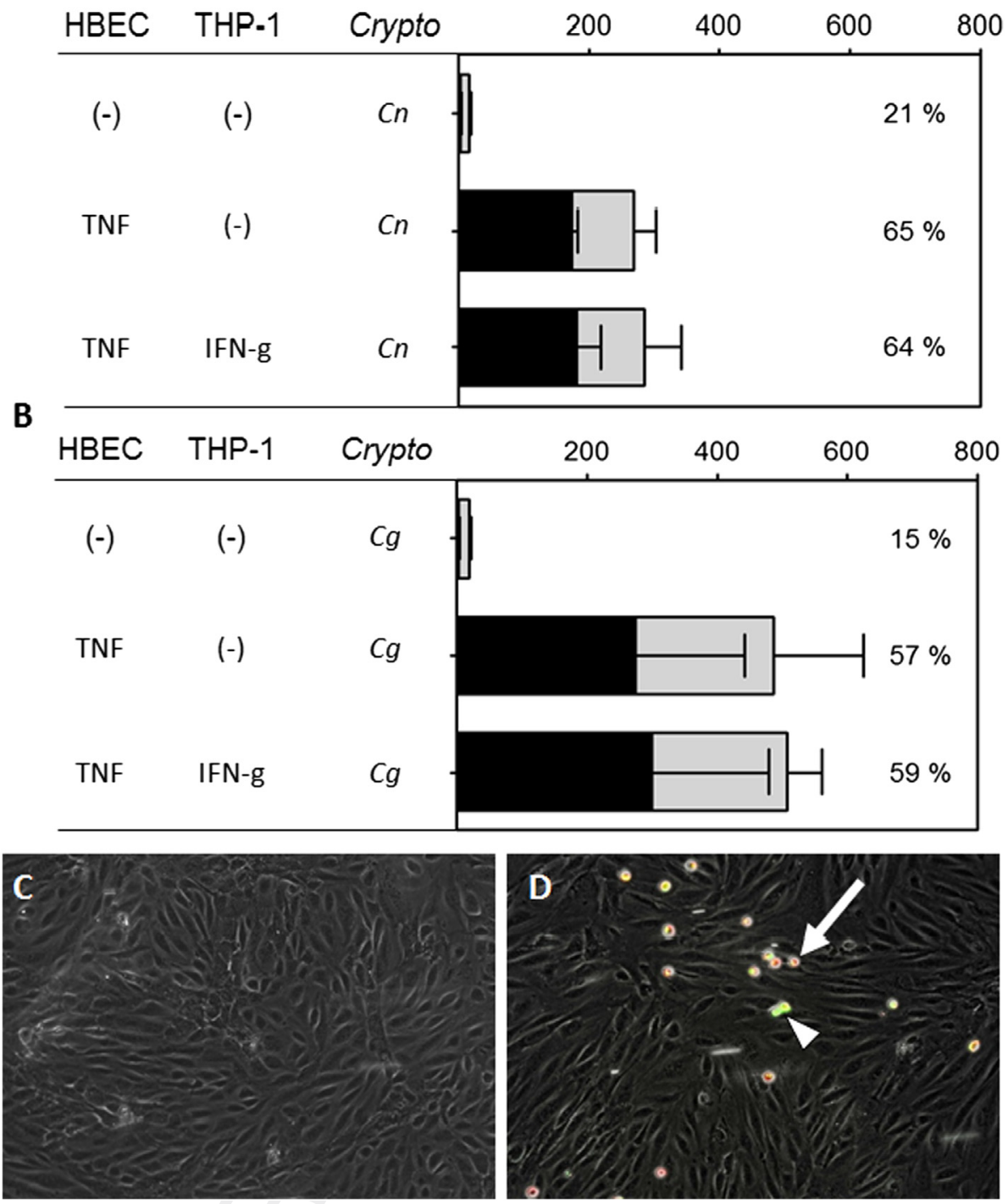

Fig. 2. Binding of THP-1 cells containing phagocytosed $\boldsymbol{C n}(\mathrm{A})$ or $\boldsymbol{C g}(\mathrm{B})$ to resting or TNF-stimulated endothelium. Grey histograms represent THP-1 cells without $C n$ or $C g$, while black histograms represent THP-1 cells containing phagocytosed $C n$ or $C g$ (stained green). Together, the grey and black histograms represent the total number of bound THP-1 cells. Since there was no significant difference between the binding of non-stimulated and IFN- $\gamma$-stimulated THP1 cells (pre-loaded with either $C n$ or $C g$ ) to resting endothelium, only binding of non-stimulated THP-1 cells to resting HBEC is shown. The proportion of THP1 cells associated with cryptococci under each condition is indicated by the percentage. FOV: field of view, $200 \times$ magnification. C and D: representative images of the binding of non-stimulated THP-1 cells without internalized cryptococci (labelled red with WGA, arrow) or containing phagocytosed $\mathrm{Cn}$ (appear green due to FITC labelling of the internalized cryptococci, arrowhead) on non-stimulated (C) and TNF-stimulated HBEC (D), under flow conditions. Three separate experiments were performed.

histogram) and 1779 (23\%) were Uvitex-positive (grey histogram). In the case of $C g, 111,959 \pm 23,350$ THP-1 had transmigrated after $24 \mathrm{~h}$, with $8383(6.7 \%)$ of them containing Cg (Fig. 4A). Among these, 3870 THP-1 (46\%) had transmigrated within THP-1 cells i.e. were Uvitex-negative (black histogram) and 4513 (54\%) were Uvitex-positive (grey histogram). As shown in Fig. $4 \mathrm{~A}$, both $C n$ and $C g$ were transported across the BBB by the Trojan horse mechanism. Although a relatively small percentage of THP-1 cells contained intracellular cryptococci, the number of $\mathrm{Cn}$ transported by this route was significantly greater than that of $\mathrm{Cg}$. The number of free cryptococci in the baso-lateral chamber of the transwells is shown in Fig. 4B. The number of FITC-positive,
Uvitex-negative $\mathrm{Cn}$ (which must have been expelled from THP-1 cells after transmigration) was significantly greater than that of $C g$ (compare black histograms). These $C n$ tended to be more numerous, although not significantly, than $\mathrm{Cn}$ that had transmigrated across the BBB as free or THP-1 surfacebound fungal cells (FITC-positive, Uvitex-positive).

\subsection{Effects of THP-1 cells with or without phagocytosed cryptococci on endothelial integrity}

To assess the integrity of the endothelial monolayer following interaction with THP-1 cells \pm cryptococci ( $\mathrm{Cn}$ or $\mathrm{Cg}$ ) and subsequent to binding or transmigration, we measured 
A
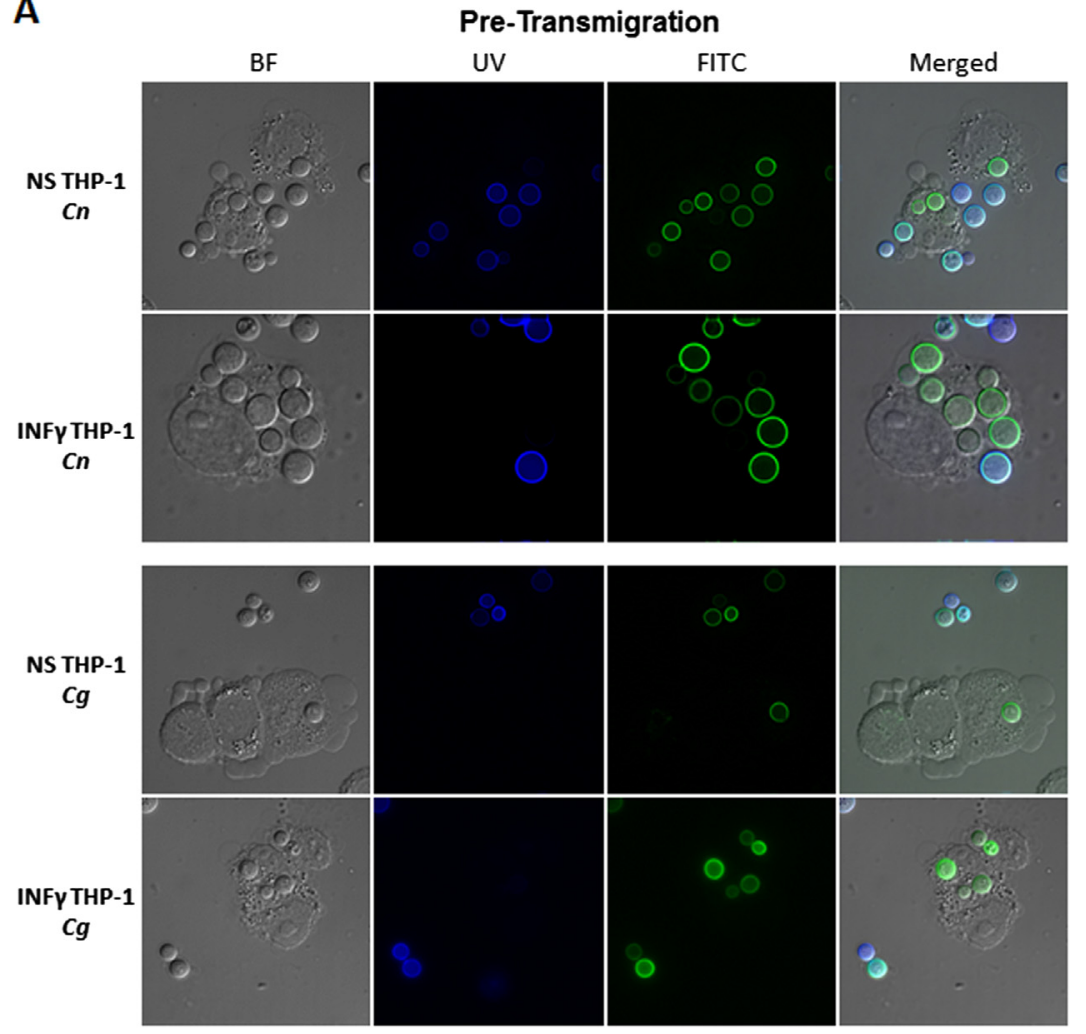

B

Post Transmigration
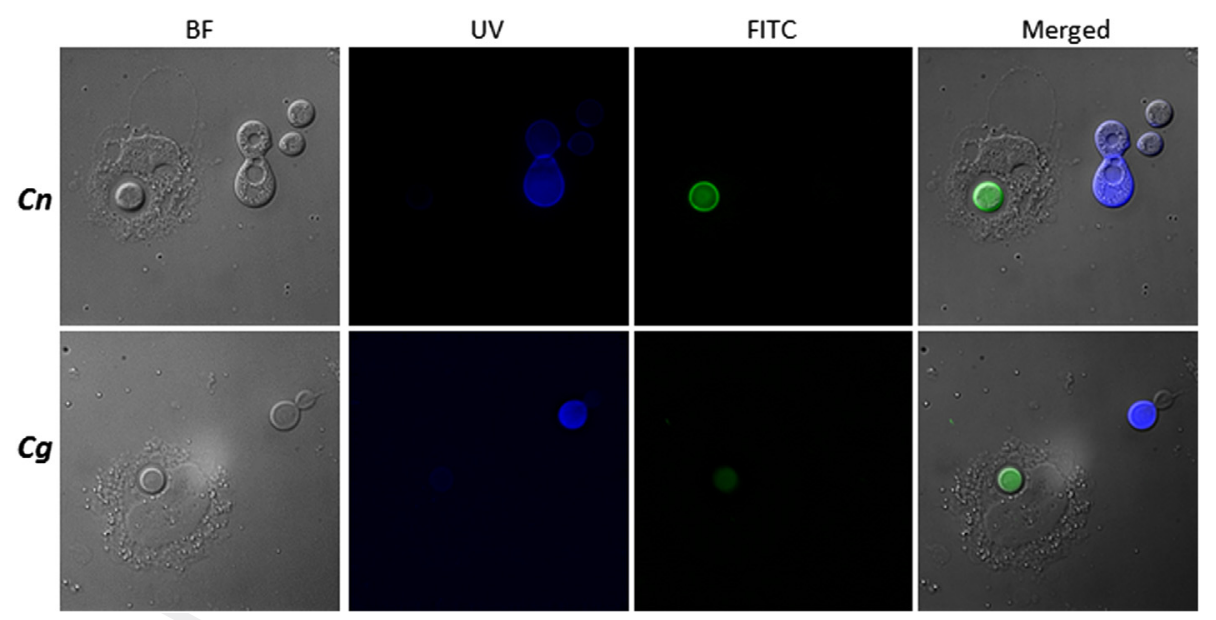

Fig. 3. Evidence for the Trojan-horse mechanism of cryptococcal transmigration through the BBB as revealed by fluorescence microscopy. (A) Phagocytosed cryptococci pre-transmigration. Following phagocytosis of FITC-labelled $C n$ or $C g$ by unstimulated and INF- $\gamma$-stimulated THP-1 cells for 3 h, unphagocytosed cryptococci were stained with Uvitex. Internalized cryptococci are observed as green/FITC + , while non-phagocytosed cryptococci are observed as both green/FITC + and blue/Uvitex + . (B) Phagocytosed cryptococci post transmigration. Following removal of non-internalized FITC $+/$ Uvitex + cryptococci postphagocytosis by two centrifugation steps over Ficoll Hypaque, THP-1 cells were incubated with HBEC monolayers for $24 \mathrm{~h}$. The colour of $C n$ or $C g$ associated with transmigrated THP-1 cells (in the basolateral chamber) was then assessed by fluorescence microscopy. Internalized FITC + cryptococci were considered to have transmigrated through the BBB as phagocytosed cells, while internalized Uvitex + cryptococci were considered to have transmigrated independently of THPIs and undergone phagocytosis post-transmigration.

the electrical impedance and permeability to FITC dextran of the HBEC monolayer, respectively (Fig. 5). Whether HBEC were co-cultured with THP-1 cells alone or THP-1 cells that had phagocytosed $\mathrm{Cn}$ or $\mathrm{Cg}$, no change to the impedance was observed over $24 \mathrm{~h}$. Similarly, impedance was not affected by stimulation of THP-1 cells with IFN- $\gamma$ or of HBEC with TNF. These findings indicate that binding of phagocytosed cryptococci to the HBEC monolayer did not affect its integrity. When HBEC monolayers were examined by light microscopy, no apparent cellular damage was observed (Fig. 6A). 
A
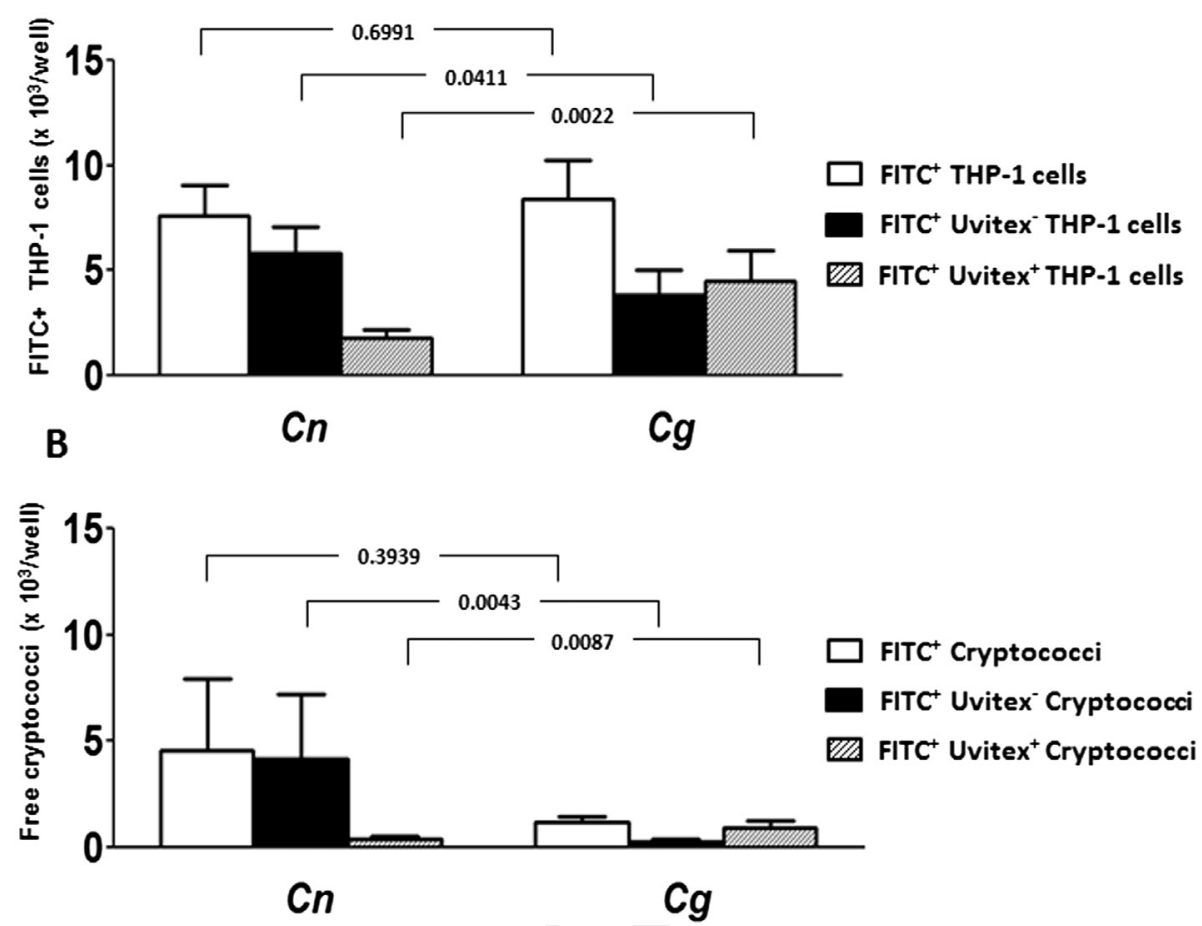

Fig. 4. Comparison of the numbers of transmigrated $C n$ or $C g$ either (A) associated with THP-1 cells or (B) as free cryptococci using fluorescence microscopy. Significantly more FITC [+] Uvitex [-] THP-1 cells containing $C n$ transmigrated across HBEC monolayers than those containing $C g$. Significantly more free $C n$ that were FITC [+] and Uvitex [-] (ie had been released from THP-1 cells post migration) were found in basal chambers than free $C g$. Three separate experiments were performed for each condition.

Permeability of HBEC monolayers to FITC-dextran was also measured in transmigration experiments, after 6 and $18 \mathrm{~h}$ of culture. No increase in permeability was noted, in contrast to that of control HBEC monolayers treated with cytochalasin B (Fig. 6B).

\section{Discussion}

This study provides further evidence for the Trojan horse mechanism of transport of cryptococci across the human brain microvascular endothelium. Using a well-established ex vivo
C. neoformans
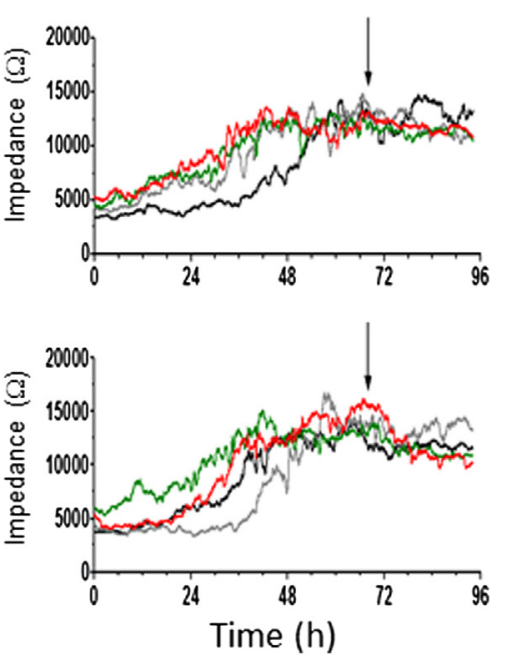

C. gattii
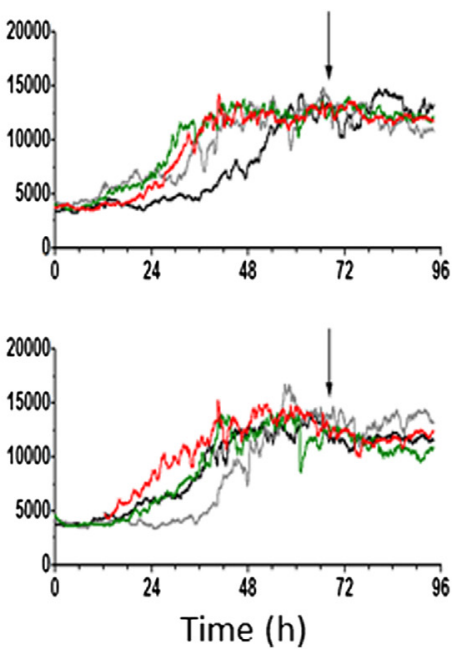
A

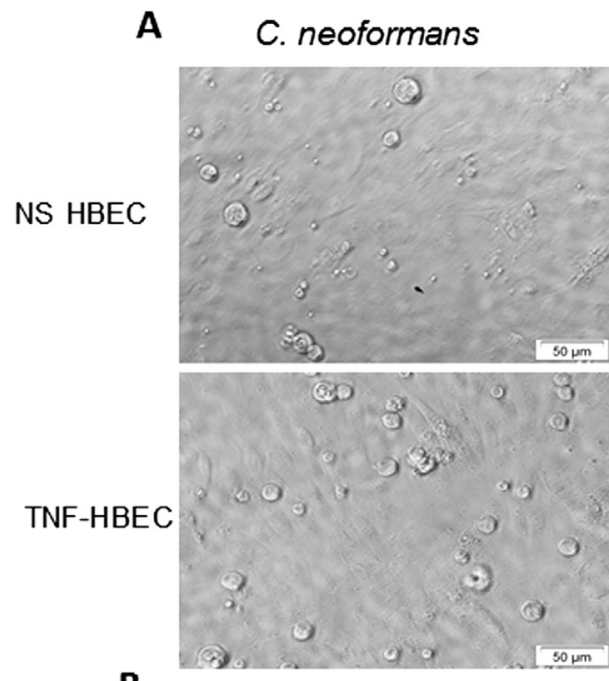

B

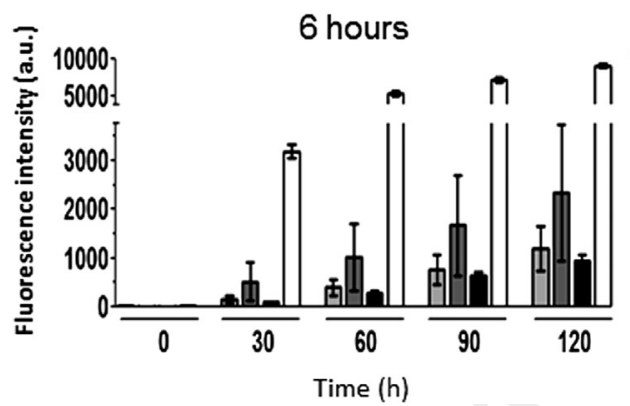

C. gattii

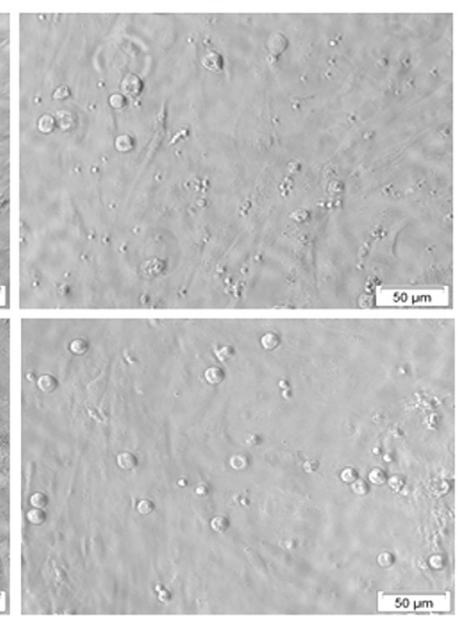

18 hours

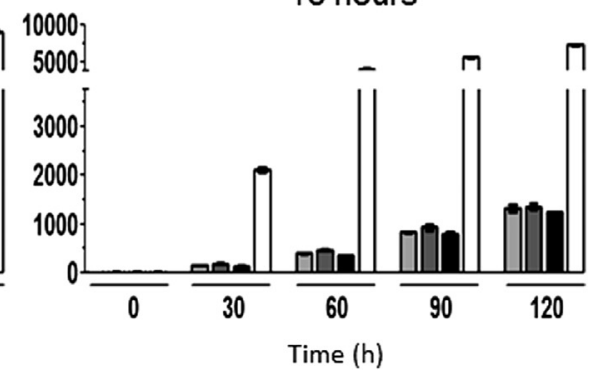

Fig. 6. Morphology (A) and permeability (B) of endothelial monolayer. In (A), images were captured $24 \mathrm{~h}$ after the addition of cryptococcus-loaded THP1 cells. In (B), resting THP-1 cells alone (light grey histograms), or with phagocytosed $C n$ (mid-grey histograms) or $C g$ (black histograms) were added for the indicated times. As a positive control for the disruption of membrane permeability, cytochalasin D were added with resting THP-1 cells (white histograms). FITCDextran $(70 \mathrm{kDa}$ ) was then added to the top chamber and the amount in the lower chamber was quantified after $6 \mathrm{~h}$ (left) and $18 \mathrm{~h}$ (right). This experiment was repeated three times.

model of BBB, recapitulated by use of an immortalized cerebral microvascular endothelial cell line, we demonstrated trans-migration of both $\mathrm{Cn}$ and $\mathrm{Cg}$ within THP-1 cells, confirming previous indirect evidence from mouse models of cryptococcal infection [5,11,12]. Importantly, electrical impedance analysis, dextran permeability studies and direct microscopy confirmed that the HBEC monolayer remained intact; i.e. its integrity was not compromised by transmigration of THP- 1 cells and cryptococci. We also observed differences in transmigration between the type strain of $\mathrm{Cn}$, H99 (genotype VNI) and the commonest, more virulent sub-genotype of $\mathrm{Cg}$ (R265, genotype VGIIa) from the Vancouver Island outbreak.

Differences in the properties of THP-1 loaded H99 and R265 in this study are of interest since early studies of $\mathrm{Cn}$ and $\mathrm{Cg}$ infection suggested that differences in clinical presentation were explained by the respective associations of $\mathrm{Cn}$ and $\mathrm{Cg}$ with host immunocompromise and immunocompetence, rather than by the causative cryptococcal species. In the study of Chen et al., $86 \%$ of patients with AIDS presented with meningitis, whereas the majority $(60 \%)$ of immune-competent patients presented with pulmonary disease and this pulmonary presentation was species-independent [2]. In the initial report of the Vancouver Island outbreak of $\mathrm{Cg}$ (genotype
VGII) infection, 57\% of HIV-negative patients presented with lung disease and $78 \%$ of HIV-infected patients presented with CNS disease [1,18]. The possibility of a genotype-dependent effect on clinical presentation was raised when Ngamskulrungroj et al. observed that strain H99 (VNI) of $C n$ and strain R265 (VGIIa) of $C g$ caused fatal brain and lung infection respectively, in a mouse inhalational model [19]. The observation by this group that a small intravenous inoculum (five cryptococcal cells) caused brain infection in only $10 \%$ of $\mathrm{Cg}$ infected mice, compared with $60 \%$ of mice infected with $\mathrm{Cn}$ [4], prompted us to study transport of these two strains across the BBB.

As expected from previous publications [20], activation of THP-1 cells with IFN- $\gamma$ resulted in a higher proportion of phagocytic THP-1 cells and higher cryptococcal loads per cell for both $C n$ and $C g$. However, $C n$ was phagocytosed by a higher proportion of unstimulated THP-1 cells than was $\mathrm{Cg}$ and loads of $C n$ per THP- 1 cell were also increased. Based on the latter observation and the report of Ngamskulrungroj et al., we predicted that adhesion of phagocytosed $\mathrm{Cn}$ to HBEC monolayers would be greater than that of $\mathrm{Cg}$. However, this was not the case. Furthermore activation of THP-1 cells by IFN- $\gamma$, a condition designed to mimic the activation status of blood monocytes during active cryptococcal infection in vivo 
[21] did not affect adherence to HBEC. Adherence to unstimulated HBEC monolayers was minimal under flow conditions (simulating the shear stress associated with blood flow through the cerebral microvasculature) but was substantially increased by pre-activation of HBEC with TNF (simulating inflammatory conditions during active infection in vivo) $[22,23]$. TNF increases the expression of adhesion molecules (such as ICAM-1, ICAM-2, and VCAM-1) on endothelial cells in cerebral capillaries and especially in post-capillary venules, which are the major site of leucocyte transmigration across the BBB in inflammatory states). TNF-induced upregulation of ICAM-1, a receptor for leucocyte integrins such as CD11b, which is expressed on monocytes (and THP1 cells), is likely explain the increased adhesion of THP-1 cells (for review, see Refs. [24,25]) Our observation that the proportion of bound THP-1 cells containing $C n$ and $C g$ was greatly increased following activation of the HBEC monolayer is of interest as it suggests that phagocytosis of cryptococci modifies THP-1 cells in a way that promotes their binding to activated endothelial cells. We can postulate that phagocytosis induces either an upregulation or-as often seen with integrins - conformational changes in THP-1 cell surface molecules, including the phagocytic receptor, adhesion and signalling molecule, CD13 ([26]. However, IFN- $\gamma$ treatment did not enhance binding to either resting or TNF-stimulated HBEC, and both CD13 ([27] and integrins are upregulated on mononuclear phagocytes by IFN- $\gamma$. Thus alternative adhesins or adhesion mechanisms must be involved; these may be associated with the release of mediators or membrane microparticles triggered by phagcocytosis. Further investigation of the mechanism of adhesion was beyond the scope of this study.

Although binding of THP-1 cells, whether or not they contained cryptococci or were activated with IFN- $\gamma$, was low in the absence of TNF-induced activation of HBEC, transmigration was unaffected by activation of either cell type, confirming that these events are independent (for review see Ley et al.). These findings suggest that transmigration proceeds by a process independent of binding to receptors upregulated on THP- 1 cells by IFN- $\gamma$ and on HBEC by TNF. This interpretation is supported by our observation that a high proportion (65\% and 57\%) of THP-1 cells that adhered to TNF-stimulated HBEC contained intracellular $\mathrm{Cn}$ and $\mathrm{Cg}$ respectively, compared with $2.5 \%$ and $3.5 \%$ after transmigration. It is of interest that in studies involving "free" cryptococci, only a similar small percentage (1.5-5.5\%) actually crossed the BBB (via transcytosis) [28]. We considered the possibility that the process of non-lytic exocytosis of cryptococci [15] from THP-1 cells post-migration lowered the apparent rates of transmigration via the Trojan horse mechanism. In this context, a report that non-lytic exocytosis of H99 from non-stimulated and IFN- $\gamma$-stimulated human primary macrophages, was greater than that of the R265 strain of $C g$, is of interest [29]. Indeed free cryptococci that had crossed the HBEC monolayer within THP-1 cells were present in the basal chambers of transwells indicative of non-lytic exocytosis and significantly more $C n$ had been released than $C g$.
Our results, if confirmed in future experiments, including the use of primary human mononuclear phagocytes, might explain the observation that multiplication of, and mortality from, $\mathrm{Cn}$ in the CNS is greater than that of $\mathrm{Cg}$ [19].

In summary, this study provides further evidence that cryptococci are transported across the BBB by the Trojan horse mechanism. Increased phagocytosis and transmigration of $\mathrm{Cn}$ within unstimulated THP-1 cells across HBEC monolayers and the increased extent of non-lytic exocytosis posttransmigration all contributed to a significantly increased load of free of $\mathrm{Cn}$ compared with $\mathrm{Cg}$ in the baso-lateral chamber of the transwells. These data invite studies of species-dependent differences in BBB interactions and transmigration. If confirmed, this may provide further insight into species-dependent differences in clinical presentations cryptococcosis.

\section{Conflicts of interest}

The authors have no conflicts of interest to declare in relation to this work.

\section{Acknowledgements}

This work was funded by a project grant \#1050106 from the National Health and Medical Research Council of Australia, the Rebecca L. Cooper Foundation, and a philanthropic donation to the Marie Bashir Institute through the Sydney Medical School Foundation. TCS is a Sydney Medical School Foundation Fellow. The authors wish to thank Dr Sophie Lev for her assistance with the fluorescence microscopy and its interpretation.

\section{References}

[1] Galanis E, Hoang L, Kibsey P, Morshed M, Phillips P. Clinical presentation, diagnosis and management of Cryptococcus gattii cases: lessons learned from British Columbia. Can J Infect Dis Med Microbiol 2009;20:23-8.

[2] Chen S, Sorrell T, Nimmo G, Speed B, Currie B, Ellis D, et al. Epidemiology and host- and variety-dependent characteristics of infection due to Cryptococcus neoformans in Australia and New Zealand. Australasian Cryptococcal Study Group. Clin Infect Dis 2000;31:499-508.

[3] Krockenberger MB, Malik R, Ngamskulrungroj P, Trilles L, Escandon P, Dowd S, et al. Pathogenesis of pulmonary Cryptococcus gattii infection: a rat model. Mycopathologia 2010;170:315-30.

[4] Ngamskulrungroj P, Chang Y, Sionov E, Kwon-Chung KJ. The primary target organ of Cryptococcus gattii is different from that of Cryptococcus neoformans in a murine model. MBio 2012;3.

[5] Charlier C, Chretien F, Baudrimont M, Mordelet E, Lortholary O, Dromer F. Capsule structure changes associated with Cryptococcus neoformans crossing of the blood-brain barrier. Am $J$ Pathol 2005;166:421-32.

[6] Perfect JR. Management of invasive mycoses in hematology patients: current approaches. Oncol Willist Park) 2004;18:5-14.

[7] Jong A, Wu CH, Gonzales-Gomez I, Kwon-Chung KJ, Chang YC, Tseng HK, et al. Hyaluronic acid receptor CD44 deficiency is associated with decreased Cryptococcus neoformans brain infection. J Biol Chem 2012;287:15298-306.

[8] Jong A, Wu CH, Shackleford GM, Kwon-Chung KJ, Chang YC, Ouyang $\mathrm{Y}$, et al. Involvement of human CD44 during Cryptococcus 
neoformans infection of brain microvascular endothelial cells. Cell Microbiol 2008;10:1313-26.

[9] Shi M, Li SS, Zheng C, Jones GJ, Kim KS, Zhou H, et al. Real-time imaging of trapping and urease-dependent transmigration of Cryptococcus neoformans in mouse brain. J Clin Invest 2010;120:1683-93.

[10] Olszewski MA, Noverr MC, Chen GH, Toews GB, Cox GM, Perfect JR, et al. Urease expression by Cryptococcus neoformans promotes microvascular sequestration, thereby enhancing central nervous system invasion. Am J Pathol 2004;164:1761-71.

[11] Santangelo R, Zoellner H, Sorrell T, Wilson C, Donald C, Djordjevic J, et al. Role of extracellular phospholipases and mononuclear phagocytes in dissemination of cryptococcosis in a murine model. Infect Immun 2004;72:2229-39.

[12] Charlier C, Nielsen K, Daou S, Brigitte M, Chretien F, Dromer F. Evidence of a role for monocytes in dissemination and brain invasion by Cryptococcus neoformans. Infect Immun 2009;77:120-7.

[13] Wang H, Sun J, Goldstein H. Human immunodeficiency virus type 1 infection increases the in vivo capacity of peripheral monocytes to cross the blood-brain barrier into the brain and the in vivo sensitivity of the blood-brain barrier to disruption by lipopolysaccharide. J Virol 2008;82:7591-600.

[14] Ma H, Croudace JE, Lammas DA, May RC. Expulsion of live pathogenic yeast by macrophages. Curr Biol 2006;16:2156-60.

[15] Alvarez M, Casadevall A. Phagosome extrusion and host-cell survival after Cryptococcus neoformans phagocytosis by macrophages. Curr Biol 2006; $16: 2161-5$.

[16] Weksler BB, Subileau EA, Perriere N, Charneau P, Holloway K, Leveque $\mathrm{M}$, et al. Blood-brain barrier-specific properties of a human adult brain endothelial cell line. FASEB J 2005;19:1872-4.

[17] Wen B, Combes V, Bonhoure A, Weksler BB, Couraud PO, Grau GE. Endotoxin-induced monocytic microparticles have contrasting effects on endothelial inflammatory responses. PLoS One 2014;9:e91597.

[18] MacDougall DS. A dearth of groundbreaking data at 14th international conclave of HIV medical community. IAPAC Mon 2002;8:276-82.
[19] Ngamskulrungroj P, Price J, Sorrell T, Perfect JR, Meyer W. Cryptococcus gattii virulence composite: candidate genes revealed by microarray analysis of high and less virulent Vancouver island outbreak strains. PLoS One 2011;6:e16076.

[20] Cheng PY, Sham A, Kronstad JW. Cryptococcus gattii isolates from the British Columbia cryptococcosis outbreak induce less protective inflammation in a murine model of infection than Cryptococcus neoformans. Infect Immun 2009;77:4284-94.

[21] Zhou Q, Murphy WJ. Immune response and immunotherapy to Cryptococcus infections. Immunol Res 2006;35:191-208.

[22] Grau GE, Lou J. TNF in vascular pathology: the importance of plateletendothelium interactions. Res Immunol 1993;144:355-63.

[23] Pober JS. Immunobiology of human vascular endothelium. Immunol Res 1999;19:225-32.

[24] Ronald JA, Ionescu CV, Rogers KA, Sandig M. Differential regulation of transendothelial migration of THP-1 cells by ICAM-1/LFA-1 and VCAM-1/VLA-4. J Leukoc Biol 2001;70:601-9.

[25] Ley K, Laudanna C, Cybulsky MI, Nourshargh S. Getting to the site of inflammation: the leukocyte adhesion cascade updated. Nat Rev Immunol 2007;7:678-89.

[26] Licona-Limon I, Garay-Canales CA, Munoz-Paleta O, Ortega E. CD13 mediates phagocytosis in human monocytic cells. J Leukoc Biol 2015;98:85-98.

[27] Gabrilovac J, Cupic B, Zivkovic E, Horvat L, Majhen D. Expression, regulation and functional activities of aminopeptidase $\mathrm{N}$ (EC 3.4.11.2; APN; CD13) on murine macrophage $\mathbf{J} 774$ cell line. Immunobiology 2011;216:132-44.

[28] Maruvada R, Zhu L, Pearce D, Zheng Y, Perfect J, Kwon-Chung KJ, et al. Cryptococcus neoformans phospholipase B1 activates host cell Rac1 for traversal across the blood-brain barrier. Cell Microbiol 2012;14:1544-53.

[29] Voelz K, Lammas DA, May RC. Cytokine signaling regulates the outcome of intracellular macrophage parasitism by Cryptococcus neoformans. Infect Immun 2009;77:3450-7. 